\title{
Ajuste de modelos matemáticos à biomassa seca dos compartimentos de plantas lenhosas em área de caatinga
}

\author{
Ajustment of mathematical models dry biomass \\ of laxy plant compartiments in caatinga área
}

\author{
Thyego Nunes Alves Barreto1, José Antônio Aleixo da Silva², \\ Rinaldo Luiz Caraciolo Ferreira ${ }^{3}$ e Cleuma Christir da Silva Almeida ${ }^{4}$
}

\begin{abstract}
Resumo
O trabalho vem com objetivo de gerar equações locais para estimar a fitomassa aérea total e dos compartimentos de plantas lenhosas na Caatinga. A pesquisa foi realizada em área de Caatinga arbustivo-arbórea, no município de Floresta - PE. Para o inventário florestal foram instaladas 35 unidades amostrais de $20 \mathrm{~m} \times 20 \mathrm{~m}$ distadas entre si $80 \mathrm{~m}$ e das bordas $50 \mathrm{~m}$, onde todas as plantas com diâmetro a altura do peito (DAP $\geq 1,9 \mathrm{~cm}$ ) foram mensuradas e submetidas a analises florísticas e fitossociológicas apenas para identificar as 5 espécies de maior $\mathrm{VI}$, em sequência as mesmas foram distribuídas em 5 classes diamétricas, sendo escolhidos 8 indivíduos por classe, totalizando 200 exemplares arbustivo-arbóreos para coleta de biomassa pelo método destrutivo. De posse desses dados foram ajustados modelos matemáticos por parte da planta e para o total. De acordo com os resultados foi possível gerar equações para biomassa seca (kg) por indivíduo arbóreo com $\mathrm{R}^{2}=0,86, \mathrm{~S}(\mathrm{xy}) \%=26,0 \%$ e para os compartimentos: Fuste, Galho grosso e Galho fino, valores de $\mathrm{R}^{2}=0,89 ; 0,71$ e 0,72 nesta ordem, com erros percentuais de $22,3 \% ; 54 \%$ e $35 \%$ respectivamente, sendo os modelos que melhor se ajustaram, Schumacher-Hall e Gompertz. Conclui-se que foi possível gerar equações confiáveis por parte da planta e para sua biomassa total com boa precisão em áreas de Caatinga.
\end{abstract}

Palavras-chave: Ajuste de equações; Fitomassa; Floresta Seca.

\begin{abstract}
The work aims to generate local equations to estimate the total aerial biomass and compartments of woody plants in the Caatinga. A research carried out in the Shrub-tree, Caatinga area, in the municipality of Floresta - PE. For the forest inventory, 35 units were installed $20 \mathrm{mx} 20 \mathrm{~m}$ apart $80 \mathrm{~m}$ apart and $50 \mathrm{~m}$ edges, where all plants as diameter at breast height $(\mathrm{DAP} \geq 1.9 \mathrm{~cm})$ were measured and submitted to floristic and phytosociological analysis only to identify 5 species of higher $\mathrm{VI}$, in sequence as united were distributed in 5 diametric classes, being chosen 8 individuals per class, totalizing 200 shrub-arboreal specimens for the collection of biomass by the destructive method. Possesses timely data on mathematical models by the plant and for the total. According to the results for possible to generate equations for dry biomass $(\mathrm{kg})$ per arboreal individual with $\mathrm{R}^{2}=0.86, \mathrm{~S}(\mathrm{xy}) \%=26.0 \%$ and for the compartments: Fuste, Thick Branch and Thin Branch, values of $R^{2}=0.89 ; 0.71$ and 0.72 in order, with percentage errors of $22.3 \% ; 54 \%$ and $35 \%$ respectively, with the models that fit best, Schumacher-Hall and Gompertz. It was concluded that it was possible to generate reliable equations for the plant and for its total biomass with good accuracy in Caatinga areas.
\end{abstract}

Keywords: Adjustment of equations; Phytomass; Dry forest.

\section{INTRODUÇÃO}

O Semiárido brasileiro é considerado como a região árida mais habitada do mundo, mas a pressão antrópica sobre esse bioma vem se intensificando ao longo dos anos, acarretando na ampliação de áreas em diversos estágios de sucessão secundária, provocados por intervenções, a partir do corte indiscriminado de espécies arbóreas nativas como fonte de energia (CASTELLETTI et al., 2004; DRUMOND et al., 2008).

\footnotetext{
${ }^{1}$ Doutor em Ciências Florestais. UFRPE - Universidade Federal Rural de Pernambuco. Rua Dom Manuel de Medeiros, s/n - 52171-900 - Recife, PE, Brasil. E-mail: thyego nunes@hotmail.com.

2Professor Titular do Departamento de Ciência Florestal. UFRPE - Universidade Federal Rural de Pernambuco. Rua Dom Manuel de Medeiros, s/n - 52171-900 - Recife, PE, Brasil. E-mail: jaaleixo@gmail.com.

3Professor Associado do Departamento de Ciência Florestal. UFRPE - Universidade Federal Rural de Pernambuco. Rua Dom Manuel de Medeiros, s/n - 52171-900 - Recife, PE, Brasil. E-mail: rinaldo.ferreira@ufrpe.br.

${ }^{4}$ Mestre em Engenharia Ambiental. UFRPE - Universidade Federal Rural de Pernambuco. Rua Dom Manuel de Medeiros, s/n - Recife, PE - 52171900 - Recife, PE, Brasil. E-mail: cleumaufrpe@gmail.com.
}

Sci. For., Piracicaba, v. 46, n. 118, p. 285-295, jun. 2018 DOI: dx.doi.org/10.18671/scifor.v46n118.14 
No Bioma Caatinga, a aceleração da degradação ambiental já alterou cerca de $50 \%$ de suas áreas e apenas 1\% desse restante é protegido legalmente pelo Sistema Nacional de Unidades de Conservação (SNUC - lei 9.985/00) (CAVALCANTI; ARAÚJO, 2008).

Além do mais, vinte e cinco por cento da demanda energética da indústria, comércio e uso doméstico na região nordeste são extraídas das áreas de Caatinga, muitas vezes limitadas em produtividade, com arvoretas esgalhadas e arbustos que pouco ultrapassam os $10 \mathrm{~m}$ de altura, com biomassa aérea variando entre 20t.ha ${ }^{-1}$ e 80t.ha ${ }^{-1}$ (RIEGELHAUPT; PAREYN, 2010).

Nesse sentido, manejar adequadamente a biomassa florestal favorece a sua condição como fonte de energia renovável e proporciona o aproveitamento de resíduos de sua exploração para o provimento da floresta, por meio de informações sobre as quantidades de fitomassa arbórea por compartimento de plantas e para o total, geralmente coletadas por uma abordagem direta (destrutiva), desbastando, separando e pesando seus compartimentos individualmente.

No entanto, este método é bastante oneroso e demanda tempo para sua concretização, além de gerar um elevado dano ambiental, mesmo em áreas de manejo predispostas ao corte.

Por outro lado, o método indireto, apoiado nas relações alométricas entre a variável biomassa, de difícil obtenção, com variáveis facilmente coletadas em campo, como diâmetro e altura, sendo bastante eficaz e vantajoso, pois baseia-se no ajuste de modelos matemáticos a uma base de dados pré-disponibilizada pelo método direto, realizado em levantamentos anteriores ou inventários pré-corte, no mesmo sítio ou em áreas estruturalmente e floristicamente similares.

Deste modo, torna-se possível a geração de equações locais para dada região, capazes de estimar a biomassa seca em áreas que venham a ser inventariadas, tanto para o monitoramento, quanto para um novo plano de manejo, tornando-o mais eficiente, reduzindo os impactos, custos, e o tempo na quantificação de biomassa.

Em soma, este tipo de abordagem facilita a tomada de decisão em relação a permanência ou supressão das árvores e seus compartimentos (Fuste, galho e folha) como fonte de energia e/ou ciclagem de nutrientes para o reestabelecimento do sítio após a exploração da vegetação.

Ademais, poucos são os trabalhos desenvolvidos em áreas de Caatinga que abordaram o método indireto, principalmente os que trataram das quantidades de biomassa por compartimentos da planta (ABREU et al., 2016; DRUMOND, 2008; LIMA et al., 1996; SAMPAIO; SILVA, 2005; SILVA; SAMPAIO, 2008; ZÁKIA et al., 1992).

Neste contexto, estimar a biomassa florestal de região semiárida em pleno processo de sucessão secundária é uma alternativa para o fornecimento de informações acerca da exploração racional dos recursos florestais.

Deste modo, a pesquisa visa ajustar modelos matemáticos aos dados de biomassa aérea seca por compartimento das árvores e para o total por indivíduo arbóreo em função do DAP e altura em área de caatinga arbustiva-arbórea em sucessão secundária, afim de gerar equações gerais que facilitem as estimativas de biomassa seca por compartimento e indivíduo arbóreo na região.

\section{MATERIAL E MÉTODOS}

\section{Área de estudo}

A pesquisa foi realizada na fazenda Itapemirim, pertencente à Empresa Agrimex S.A do Grupo João Santos, no município de Floresta, em uma área com cerca de 50 ha, localizada nas proximidades das coordenadas geográficas $8^{\circ} 30^{\prime} 37^{\prime \prime} \mathrm{S}$ e $37^{\circ} 59^{\prime} 07^{\prime \prime} \mathrm{W}$, correspondente ao primeiro talhão de corte da área submetida ao Plano de Manejo Florestal.

O município está inserido na Depressão Sertaneja, com relevo predominantemente suave-ondulado, (CPRM, 2005), com Luvissolo Crômico pouco profundo de textura superficial arenosa (EMBRAPA, 2001).

A vegetação é composta por Caatinga Hiperxerófila com Floresta Caducifólia. O clima segundo a classificação de Köppen, é do tipo BSh, Tropical Semiárido com chuvas de verão. O período chuvoso vai de novembro a abril, com precipitação média anual de $432 \mathrm{~mm}$ e temperatura média anual de $26^{\circ} \mathrm{C}$ (CPRM, 2005).

\section{Coleta e Análise dados}

Inicialmente realizou-se amostragem sistemático com 35 parcelas de $400 \mathrm{~m}^{2}(20 \mathrm{~m} \mathrm{X} 20 \mathrm{~m})$ cada, sendo distadas entre si $80 \mathrm{~m}$ e das bordas do talhão $50 \mathrm{~m}$ e georeferenciadas com um GPS 
(Timber navigation). Os indivíduos arbóreos, bem como suas bifurcações foram mensurados em cada parcela, com circunferência medida a $1,30 \mathrm{~m}$ do solo (DAP $\geq 1,9 \mathrm{~cm}$ ), conforme as especificações do Comitê Técnico Científico da Rede de Manejo Florestal da Caatinga (CPRM, 2005).

Em seguida, foram realizados os levantamentos florísticos e as estimativas dos parâmetros fitossociológicos da distribuição horizontal (densidade absoluta e relativa, dominância absoluta e relativa, frequência, frequência absoluta e relativa, valor de importância) para reconhecer as 5 espécies com maior VI (valor de importância), conforme as fórmulas de Braun-Blanquet (1932) e MuellerDombois e Ellenberg (1974).

Foram selecionadas as 5 espécies com os maiores valores de VI, e para a coleta da biomassa verde em campo foram selecionados 8 indivíduos arbóreos/espécie em cada uma das cinco classes diamétricas (classe 1: amplitude de 1,9 - 3,5 cm de dap; classe 2: 3,5 - 5,1; classe 3: 5,1 - 6,7; classe 4: $6,7-8,3 \mathrm{~cm}$; classe $5: \geq 8,3)$ de diâmetro a altura à 1,30 $\mathrm{m}$ do solo (DAP $(\mathrm{cm})$ ) para as cinco espécies, total de 40 indivíduos para cada, total de 200 indivíduos amostrados.

A escolha dos indivíduos de cada uma das cinco espécies foi feita de forma aleatória dentro ou próximo das unidades amostrais, evitando-se plantas parcialmente cortadas, queimadas ou tombadas.

O processo de determinação da biomassa verde em campo foi realizado pelo método destrutivo separando os compartimentos da planta em: fuste, galho grosso (diâmetro na base do galho $\geq 1,9 \mathrm{~cm}$ ), galho fino (diâmetro nas duas bases do galho $\leq 1,9 \mathrm{~cm}$ ) e folhas para cada árvore-amostra e pesados.

Para a determinação do peso seco, amostras de 100 a 300g de cada compartimento em campo foram extraídas e conduzidas a uma estufa de circulação forçada de ar à temperatura de $70^{\circ} \mathrm{C}$ até a estabilização de seus respectivos pesos secos.

$$
B s=\frac{P u(c) \cdot P s(a)}{P u(a)}
$$

Em que: $B S=$ Biomassa seca total por compartimento $(\mathrm{Kg}) ; P u(c)=$ peso úmido total no campo por compartimento $(\mathrm{Kg}) ; \operatorname{Ps}(a)=$ peso seco da amostra por compartimento $(\mathrm{kg}) ; \mathrm{Pu}(a)=$ peso úmido da amostra por compartimento $(\mathrm{Kg})$.

Foi quantificada a biomassa seca total em (Kg) por indivíduo, somando os valores de biomassa seca por compartimento, para analisar quantitativamente a biomassa seca para os compartimentos da planta e para o total, em função das classes diamétricas por espécies de maior VI. Em seguida, houve a preparação para a modelagem matemática dos dados de biomassa seca dos compartimentos, onde foram submetidos a transformação de Box-Cox, (BOX; COX, 1964), após encontrado o valor adequado de $\lambda$ foi feita a transformação dos dados verificando-se a normalidade dos mesmos pelo teste de Kolmogorov-Smirnov.

Na sequência fez-se a ANOVA, o pós-teste de Tukey (5\%) de probabilidade para definição dos grupos a serem modelados. Após essa etapa houve o ajuste de modelos matemáticos aos dados que geraram equações para estimar a biomassa seca (Kg), por compartimentos e para o total. Os modelos matemáticos não lineares e intrinsicamente lineares foram ajustados, por intermédio do algoritmo de Levenberg-Marquardt que utilizou o Método dos Mínimos Quadrados Não Lineares (SILVA et al., 2009) (tabela 1).

Tabela 1. Modelos matemáticos não lineares e intrinsicamente lineares ajustados aos dados de biomassa seca do fuste $(\mathrm{kg})$, galho grosso (diâmetro na base $\geq 1,9 \mathrm{~cm}$ ), galho fino (diâmetro na base $\leq 1,9 \mathrm{~cm}$ ), folha $(\mathrm{kg}$ ), em função do diâmetro a altura do peito $\operatorname{DAP}(\mathrm{cm})$ e altura total $\mathrm{Ht}(\mathrm{m})$.

Table 1. Non-linear and intrinsically linear mathematical models adjusted to dry biomass (kg), thick twig (base diameter $\geq 1,9 \mathrm{~cm}$ ), fine twig (base diameter $\leq 1,9 \mathrm{~cm}$ ), leaf $\mathrm{Kg}$ ), As a function of the diameter the height of the chest dap $(\mathrm{cm})$ and total height $\mathrm{Ht}(\mathrm{m})$.

\begin{tabular}{lll}
\hline & Modelos & Autores \\
\hline 1 & $\operatorname{Ln}\left(Y_{i}\right)=\beta_{0}+\beta_{1} \cdot \operatorname{Ln}\left(X_{1_{i}}\right)+\beta_{2} \cdot \operatorname{Ln}\left(X_{2_{i}}\right)+\varepsilon_{i}$ & Schumacher e Hall (Linearizado) \\
2 & $\operatorname{Ln}\left(Y_{i}\right)=\beta_{0}+\beta_{1} \cdot \operatorname{Ln}\left(X_{1_{i}}{ }^{2} X_{2_{i}}\right)+\varepsilon_{i}$ & Spurr (Linearizado) \\
3 & $Y_{i}=\beta_{0}+\beta_{1} X_{1_{i}}+\beta_{2} X_{1_{i}}{ }^{2}+\varepsilon_{i}$ & Hohenald-Krenn \\
4 & $Y_{i}=\beta_{0} \cdot X_{1_{i}}^{\beta_{1} \cdot X_{2_{i}}{ }^{\beta_{2}} \cdot \varepsilon_{i}}$ & Schumacher e Hall \\
5 & $Y_{i}=\beta_{0} \cdot e^{\left(-e^{\left(\beta_{1}-\beta_{2} \cdot X_{1_{i}}\right)}\right)}+\varepsilon_{i}$ & Gompertz \\
\hline
\end{tabular}

Em que: $Y_{i}=$ variáveis dependentes; $\beta_{0}, \beta_{1}, \beta_{2}, \ldots, \beta_{n}=$ parâmetros dos modelos; $X_{1,}, X_{2}, \ldots, X_{n i}=$ variáveis independentes; $e_{i}=$ erro aleatório. In which: $Y_{i}=$ dependent variables; $\beta_{0}, \beta_{1}, \beta_{2}, \ldots, \beta_{n}=$ parameters of the models; ; $X_{1 i}, X_{2 i}, \ldots, X_{n i}=$ independent variables; $e_{i}=$ random error. 
Barreto et al. - Ajuste de modelos matemáticos à biomassa seca

dos compartimentos de plantas lenhosas em área de caatinga

As melhores equações para as estimativas da biomassa total e por compartimentos foi realizada com base no Erro Padrão das Estimativas, Índice de Furnival, Coeficiente de determinação ajustado e a Distribuição dos Resíduos.

\section{RESULTADOS E DISCUSSÃO}

As cinco espécies de maior VI na área, foram; Poincianella bracteosa (tul.) l.p.queiroz (Caatingueira), Commiphora leptophloeos (mart.) j.b.gillett (Imburana de cambão), Mimosa ophtalmocentra mart. ex benth (jurema de imbira), Mimosa tenuiflora (willd.) poir. (Jurema preta) e Aspidosperma pyrifolium mart. (Pereiro)

A predominância da espécie Poincianella bracteosa em áreas de Caatinga que encontram-se em pleno estágio de sucessão secundária intermediária é bastante acentuada (ALVES JÚNIOR, 2010; CABRAL et al., 2013; al., 2013; FABRICANTE et al., 2009; FERRAZ et al., 2014; MARANGON et al. 2013).

O mesmo foi observado para as espécies: Mimosa tenuiflora, Aspidosperma pyrifolium e Commiphora leptophloeos, que ao ocuparem maiores colocações em importância estrutural nessas áreas, representam um indicativo de processo de sucessão secundária intermediário (ARAÚJO et al., 2012; CABRAL et al., 2013; CALIXTO JÚNIOR; DRUMOND, 2011; COELHO et al., 2012; FERREIRA et al., 2016).

Os resultados obtidos para biomassa total seca $(\mathrm{Kg})$ dos 200 indivíduos arbóreos coletados em campo para as cinco espécies de maior VI, apresentaram os seguintes parâmetros estatísticos: média $(10,6)$, desvio padrão $(7,5)$, variância da amostra $(55,6)$, coeficiente de variação $(70,5 \%)$, erro admissível $(9,8 \%)$, erro amostral (1,04\%), intervalo de confiança $(9,5 \leq 10,6 \leq 11,6)$ e total $(2115 \mathrm{~kg})$.

Pôde-se observar que a amostragem realizada foi suficiente para a validação de estimativas da biomassa seca $(\mathrm{Kg})$ na área de pesquisa, com erro amostral inferior ao estipulado pela Instrução Normativa No 007/2006 do órgão fiscalizador do estado, Agência Estadual do Meio Ambiente (CPRH, 2006), que considera o erro de amostragem de 20\% (vinte por cento) e $90 \%$ (noventa por cento) de probabilidade.

Com relação a Análise de Variância (ANOVA) para as 5 espécies amostradas em função dos compartimentos da planta $(\mathrm{Kg})$ foi observado diferenças significativas apenas para a biomassa seca dos Galhos grossos e Galhos finos. E de forma complementar o pós-teste de Tukey a (5\%) detectou diferença significativa para a C. leptophloeos com valores de médias para o Galho grosso 1,8(kg) e Galho fino 3,3(kg), diferentes estatisticamente das demais espécies nos mesmos compartimentos, ocasionando a formação de 4 grupos, sendo dois compostos por Galho grosso e Galho fino para C. leptophloeos (40 amostras cada) e os outros dois com Galho grosso e Galho fino para outras espécies (160 amostras cada).

\section{Ajuste de Equações}

Os modelos se ajustaram melhor para as variáveis Fuste (kg) e o Total (kg), com 0,89 e 0,86 de $\mathrm{R}_{\text {aj }}^{2}$ e erros percentuais de $22 \%$ e $26 \%$ respectivamente.

Em estudos relacionados a modelagem de biomassa verde, Alves Júnior (2010) obteve como resultado equações para todas as espécies superiores a 0,90 de $\mathrm{R}^{2}$ aj para biomassa total, com erros percentuais (39\% a $152 \%)$.

Em outro estudo na mesma região, realizado por Abreu (2012), o R²aj para biomassa verde total foi de 0,78 e $S_{\mathrm{xy}}(\%)=29,67 \%$, equação pelo modelo de Schumacher-Hall (SCHUMACHER; HALL, 1939) . Modelo este que similarmente se destacou neste trabalho juntamente com as equações geradas pelo modelo de Gompertz (modelo de crescimento) representado pela equação 5. Seus coeficientes mostraram que a biomassa total cresce de forma simultânea a biomassa do fuste pelo fato do coeficiente $b_{2}$ em ambas as variáveis apresentarem o mesmo valor de 0,17 . Para o coeficiente $\mathrm{b}_{0}$ foi observado que o valor de biomassa seca total tende a estacionariedade aos $61,85(\mathrm{~kg})$ e para o fuste o mesmo ocorreu aos $24,85(\mathrm{~kg})$, sendo teoricamente o ponto assintótico da biomassa seca das plantas para as duas variáveis em função do DAP.

Os resíduos apresentados pelos compartimentos Total e Fuste revelaram breve superestimação e indício de heterocedasticidade até valores próximos aos $15 \mathrm{Kg}$ de biomassa seca total e 5kg de biomassa seca do fuste, devido a elevada variabilidade de biomassa nas primeiras classes de diâmetro em florestas nativas. Comportamento característico em algumas áreas de caatinga que tendem 
a apresentar um número muito elevado de indivíduos com diâmetros pequenos em áreas onde o estágio de sucessão secundária predomina (AMORIM et al.,2005; SANTANA; SOUTO, 2006).
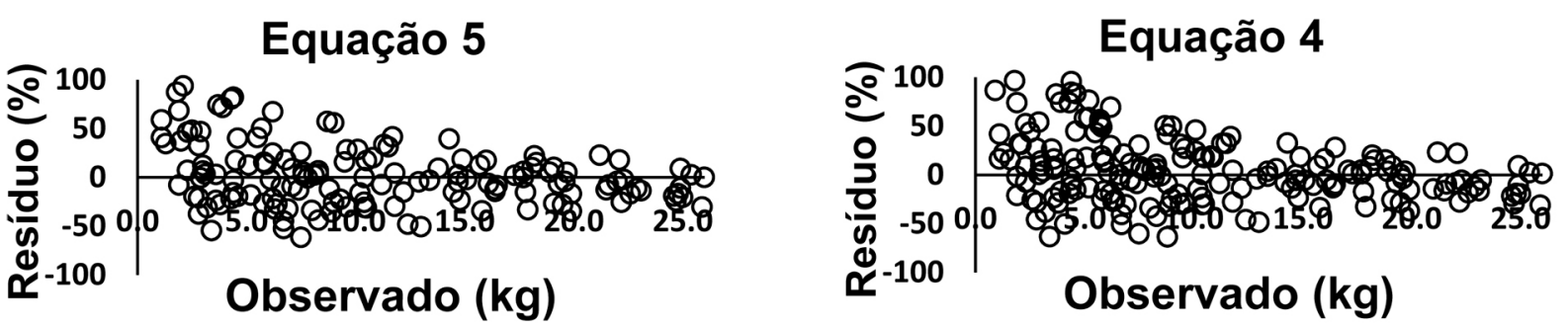

Figura 1. Gráfico dos resíduos para a biomassa seca total $(\mathrm{Kg})$, apresentado pelas equações 4 e 5 .

Figure 1. Residual graph for the total dry biomass $(\mathrm{Kg})$, presented by equations 4 and 5 .
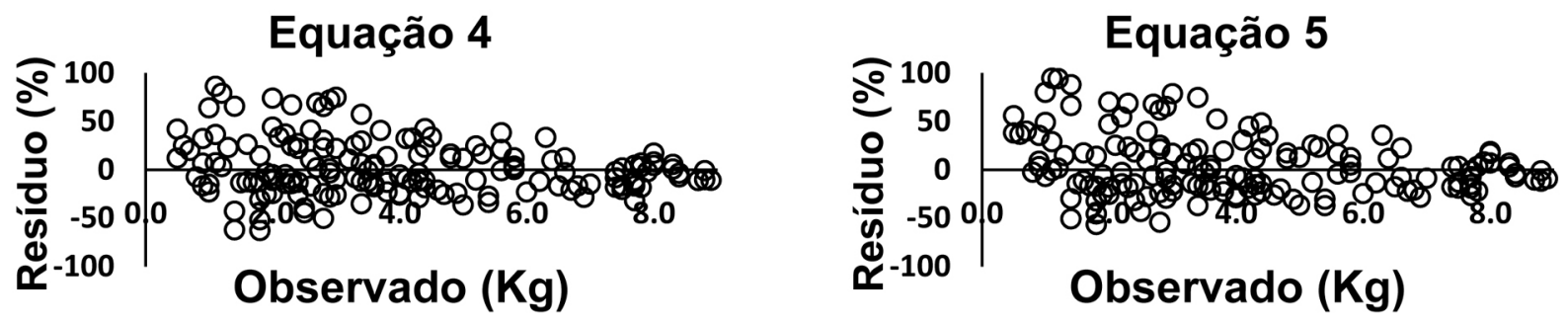

Figura 2. Gráfico dos resíduos para a biomassa seca do fuste $(\mathrm{Kg})$, apresentado pelas equações 4 e 5 .

Figure 2. Graph of the residuals for the dry biomass of the stem $(\mathrm{Kg})$, presented by equations 4 and 5 .

Para Rezende et al. (2006), essa variabilidade tende a aumentar, à medida que árvores de maior diâmetro são incluídas, mesmo aumentando a intensidade amostral.

No entanto, os resultados apresentados pelo teste de normalidade e análise gráfica dos resíduos na detecção de autocorrelação dos erros, demonstraram que as equações geradas pelos modelos de Schumacher-Hall e Gompertz foram capazes de realizar estimativas sem tendência e com variância constante na maior parte dos dados, produzindo estimativas confiáveis da biomassa seca para o total e o fuste apresentados nas Figuras ( 3 e 4 ).
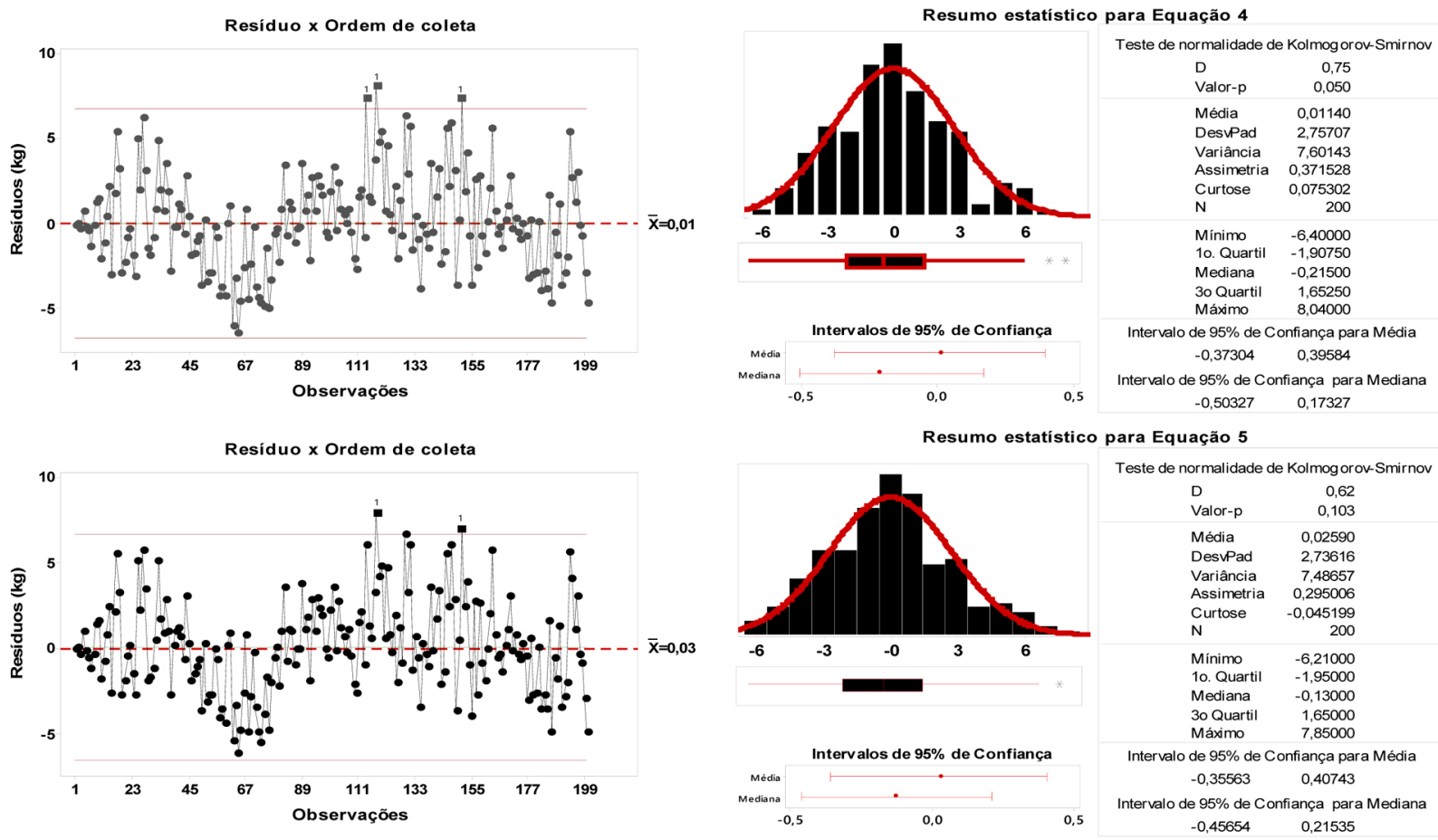

Figura 3. Ilustração do gráfico de resíduos $x$ ordem de coleta, histograma dos resíduos com ajuste de curva normal e sumário estatístico contendo o valor-p para o teste de normalidade de Kolmogorov-Smirnov da biomassa total seca para as equações 4 e 5 .

Figure 3. Illustration of waste chart $x$ collection order, histogram of residuals with normal curve fit and statistical summary containing the $\mathrm{p}$-value for the Kolmogorov-Smirnov normality test of the total dry biomass for equations 4 and 5 . 

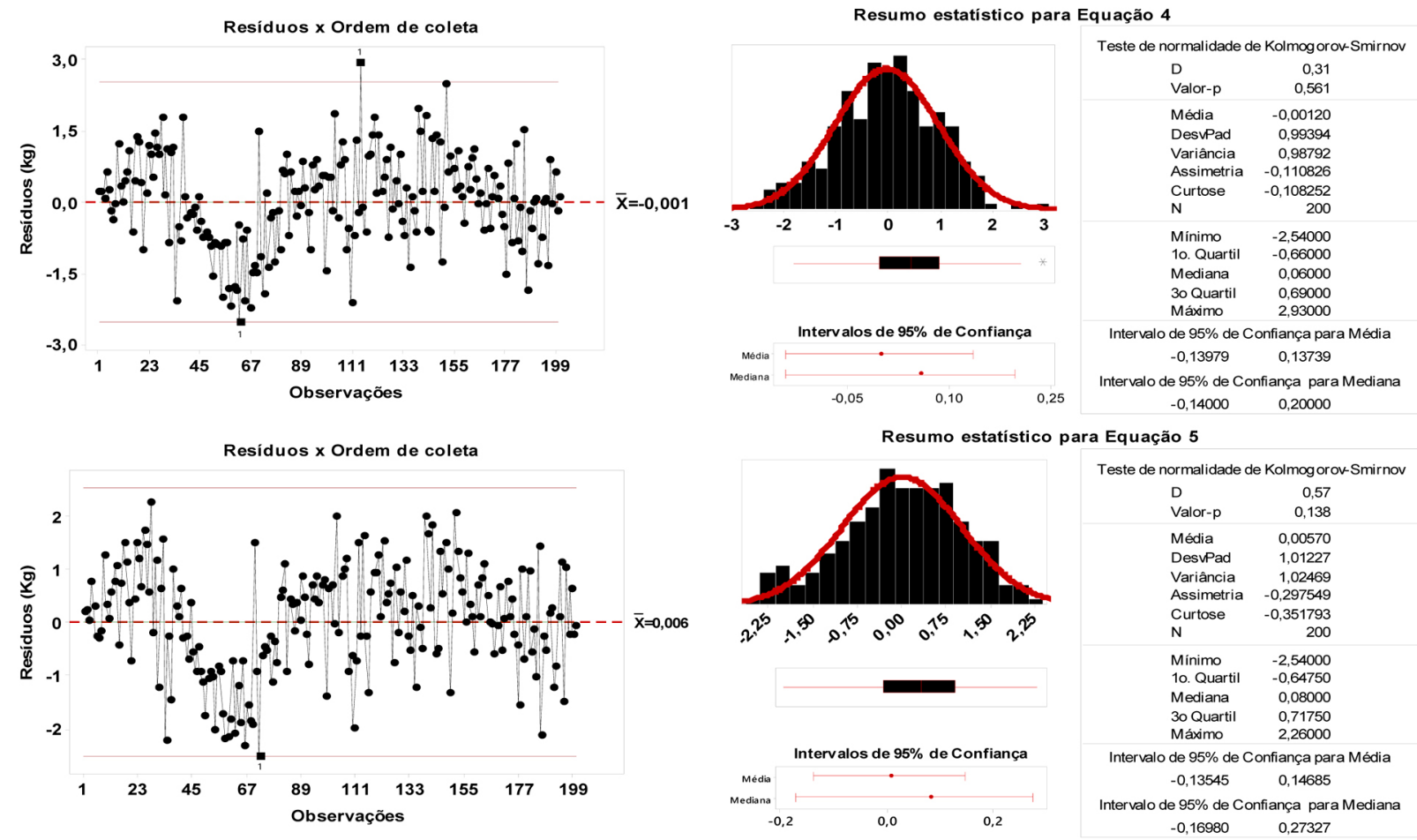

Figura 4. Ilustração do gráfico de resíduos $x$ ordem de coleta, histograma dos resíduos com ajuste de curva normal e sumário estatístico contendo o valor-p para o teste de normalidade de Kolmogorov-Smirnov da biomassa seca do fuste das equações 4 e 5.

Figure 4. Illustration of waste chart $x$ collection order, histogram of residuals with normal curve fit and statistical summary containing the $\mathrm{p}$-value for Kolmogorov-Smirnov normality test of the dry biomass of the shaft of equations 4 and 5 .

Para os compartimentos, Galho fino + Folhas (kg), Galho fino sem C. leptophloeos (kg) e Galho grosso sem C. leptophloeos $(\mathrm{kg})$ todos os modelos se ajustaram com valores de $\mathrm{R}^{2}{ }_{\text {aj }}$ acima de 0,7, porém com erros maiores que os apresentados para a biomassa total e para o fuste.

De acordo com a análise gráfica dos resíduos, a equação 4 selecionada para estimativa das variáveis Galho fino + Folhas (kg) e Galho grosso sem C. leptophloeos (kg), apresentou homogeneidade dos resíduos melhor para o primeiro compartimento se comparado ao segundo. Já para o Galho fino sem C. leptophloeos as equações 1 e 2 apresentaram uma boa distribuição percentual dos resíduos, com erros aceitáveis para a Caatinga, uma vez que poucos estudos detalharam os resíduos gerados pelo ajuste de modelos de biomassa nessa região, principalmente averiguando a normalidade e autocorrelação dos mesmos, conforme às Figuras ( 5 e 6 ).

O resíduo de ambas as variáveis não apresentaram autocorrelação, entretanto a variável Galho grosso sem C. leptophloeos (kg) não apresentou normalidade, possivelmente pelo valor de erro percentual igual a 54\% para a mesma, além da ausência de valores de biomassa desse componente nas duas primeiras classes diamétricas, por conta da fase inicial de crescimento em que muitos indivíduos amostrados se encontravam apresentando seus galhos abaixo do limite mínimo de inclusão para Galho grosso. No entanto, para o compartimento Galhos finos sem C. leptophloeos (Kg) as estimativas foram muito melhores com erros percentuais em torno dos $36 \%$ para as equações 1 e 2 , além de resíduos bem mais uniformes distribuídos de forma normal e sem evidências de autocorrelação residual (figura 8).

As equações para estimar a biomassa seca dos Galhos grossos e Galhos finos da C. leptophloeos e para as Folhas, com $\mathrm{R}^{2}$ (ai) menores que 0,7 e erros percentuais acima $40 \%$, não foram consideradas. A falta de ajuste para biomassa das folhas se deu pela variabilidade intra e interespecífica desse componente principalmente nos indivíduos jovens coletados em campo, com poucas folhas, como observado em campo, bem como a deciduidade predominante na região do estudo, que aumentou consideravelmente a variância desse compartimento.

Quanto aos Galhos grossos e finos da C. leptophloeos, a falta de ajuste foi consequência da morfologia da espécie que apresentou poucas ramificações. 


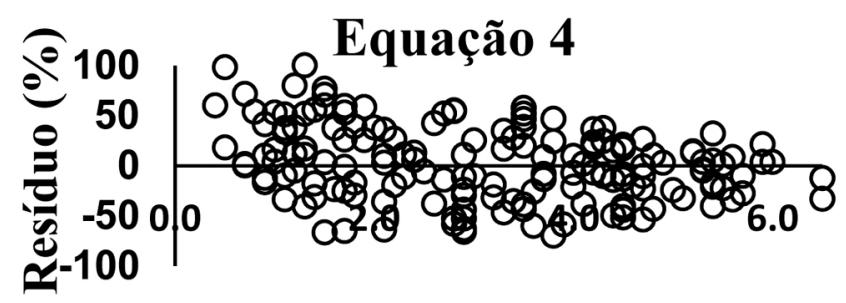

\section{Observado (Kg)}
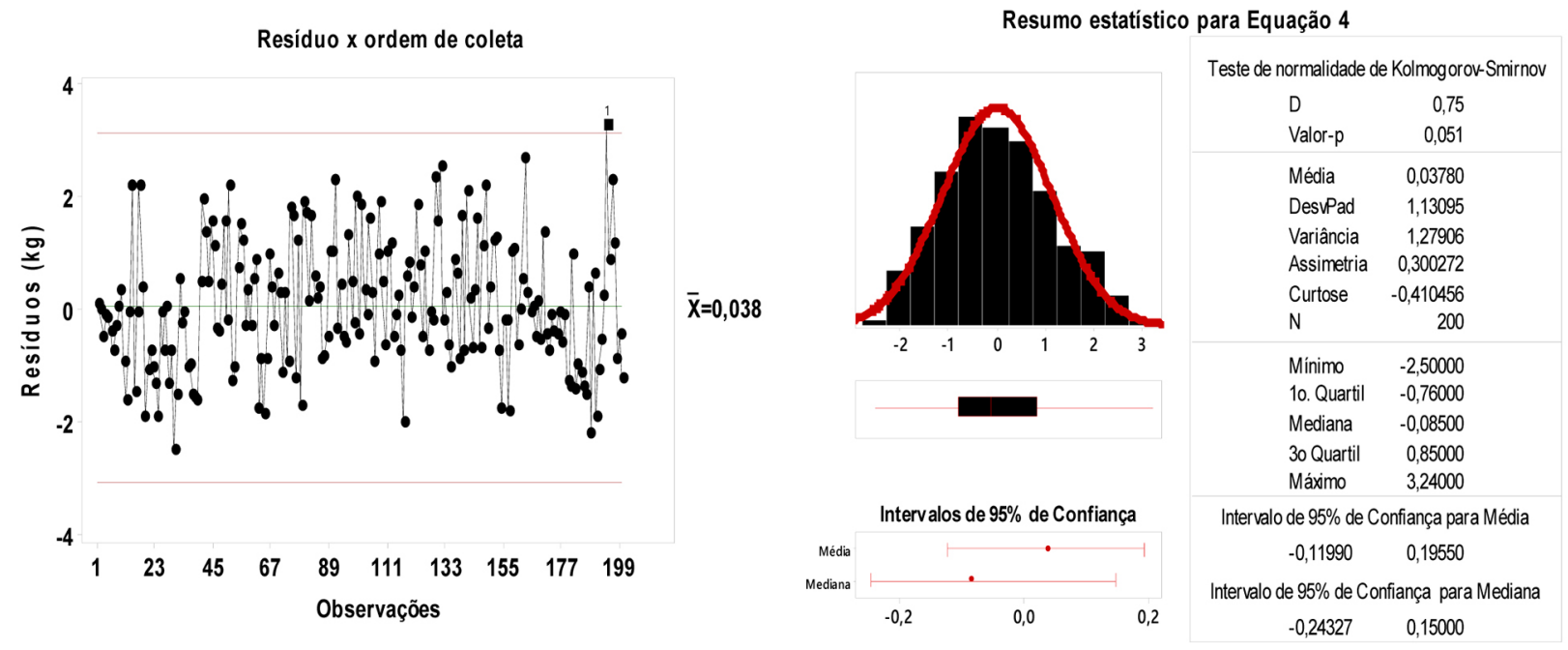

Figura 5. Resíduos em porcentagem; resíduos x ordem de coleta; histograma dos resíduos com ajuste de curva normal para o teste de normalidade de Kolmogorov-Smirnov da biomassa seca do Galho fino + Folhas (kg) para a equação 4.

Figure 5. Residuals in percentage; Waste $x$ collection order; Histogram of the residuals with normal curve fit for the Kolmogorov-Smirnov normality test of the thin branch biomass + Leaves $(\mathrm{kg})$ for equation 4.

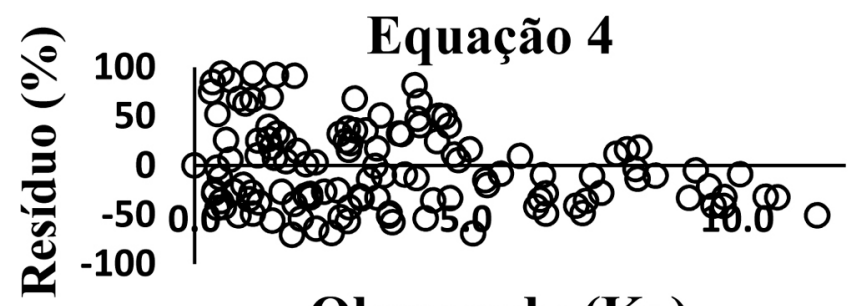

\section{Observado (Kg)}
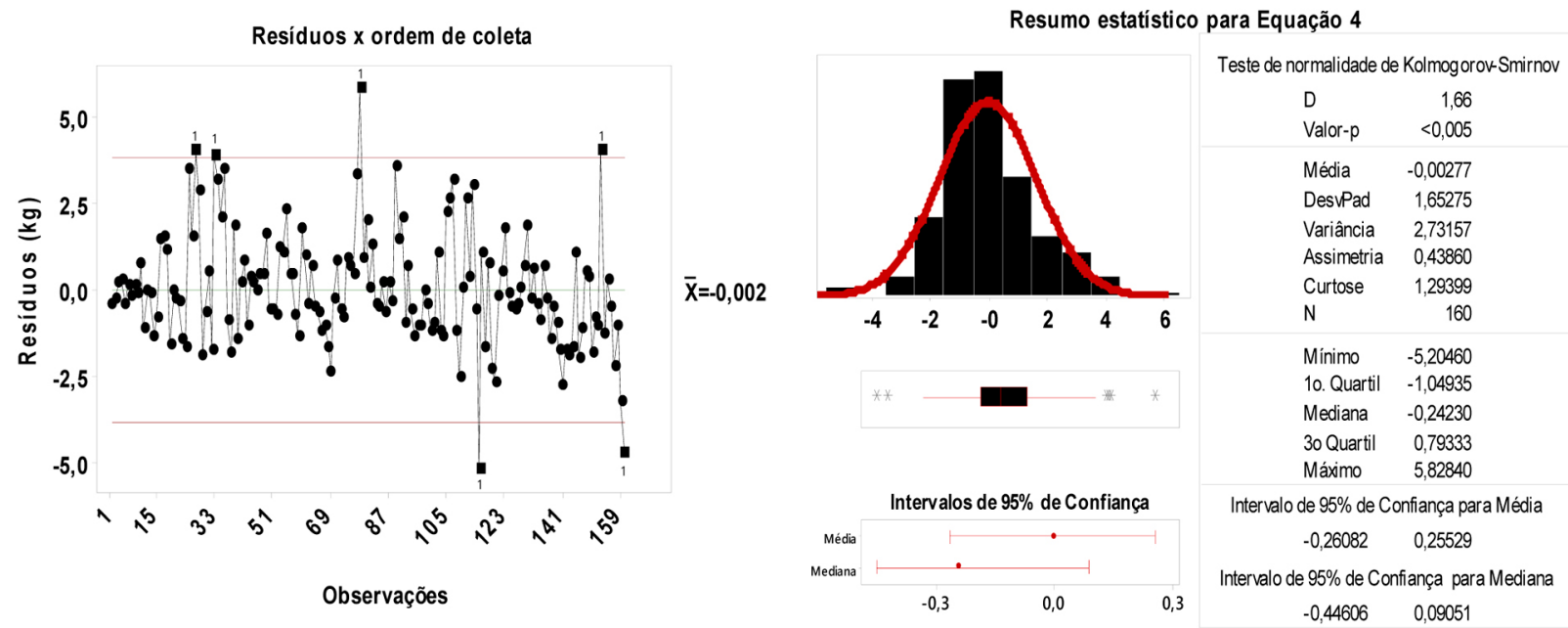

Figura 6. Resíduos em porcentagem; resíduos $x$ ordem de coleta; histograma dos resíduos com ajuste de curva normal para o teste de normalidade de Kolmogorov-Smirnov da biomassa seca do Galho grosso sem $C$. leptophloeos $(\mathrm{kg})$ para a equação 4.

Figure 6. Waste in percentage; Waste $x$ collection order; Histogram of the residuals with normal curve fit for the Kolmogorov-Smirnov normality test of the dry thick-walled biomass without C. leptophloeos (kg) for equation 4. 

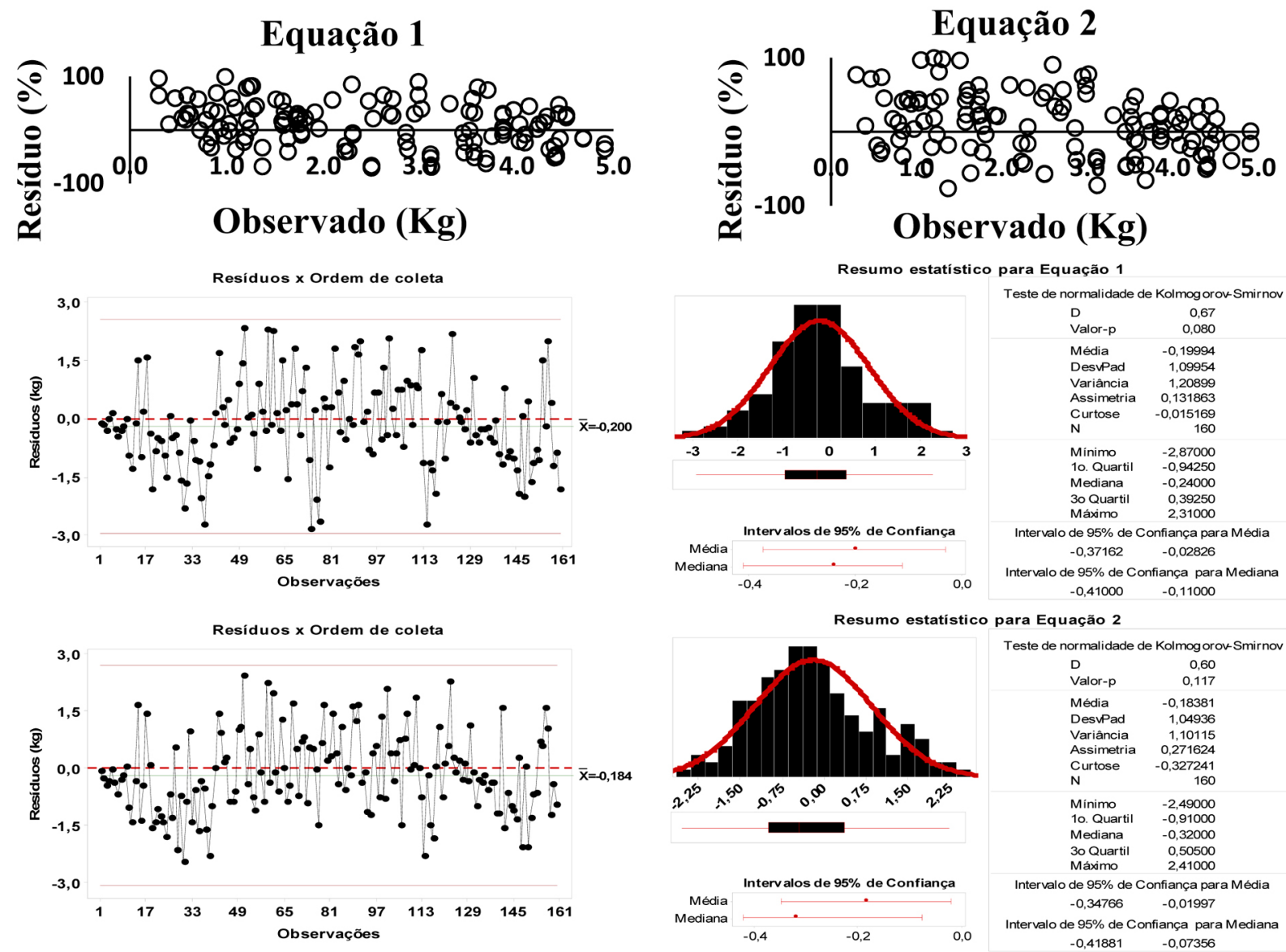

Figura 7. Resíduos em porcentagem; resíduos $x$ ordem de coleta, histograma dos resíduos com ajuste de curva normal e sumário estatístico contendo o valor-p para o teste de normalidade de Kolmogorov-Smirnov dos Galhos finos sem C. leptophloeos $(\mathrm{Kg})$ para as equações 1 e 2.

Figure 7. Waste in percentage; Residuals $x$ order of collection, histogram of the residuals with normal curve fit and statistical summary containing the p-value for the Kolmogorov-Smirnov normality test of the Thin branches without $C$. leptophloeos $(\mathrm{Kg})$ for equations 1 and 2 .

Foi possível gerar equações para a biomassa total, fuste, Galhos finos sem a presença da C. leptophloeos e Galhos finos + folhas para todas as espécies representantes na área da pesquisa.

A evidência de baixa correlação existente entre à altura e a biomassa dos compartimentos está relacionada a estatura uniforme das árvores da região árida de Pernambuco, com baixa precipitação média anual e abundante presença de solos rasos e arenosos que influem diretamente no porte das plantas dessas regiões (GIULIETTI et al., 2004; LEAL et al., 2005).

Apesar da variação nas alturas das árvores da região serem pequenas, as quantidades de biomassa são bastante variáveis, o que acarretou na baixa correlação entre as massas das plantas e suas respectivas alturas. Diferentemente da variável dap $(\mathrm{cm})$ que apresentou boas correlações nos trabalhos realizados Caatinga (SAMPAIO; SILVA, 2005; SILVA; SAMPAIO, 2008).

Os valores de $\mathrm{S}_{\mathrm{xy}}(\%)(22,3 \%-37,8 \%)$ podem ser considerados razoáveis, pois de acordo com Rezende et al. (2006) a maioria dos erros encontrados nas equações selecionadas para estimar biomassa em florestas nativas estão entre 25 e 40,7\%. Para Santos et al. (2009) ajustar modelos matemáticos aos dados de biomassa em espécies nativas geralmente apresentam erros elevados, por conta de suas características típicas em termos de arquitetura dos fustes. Sobretudo, estes erros retratam a variabilidade real (BASUKI et al., 2009; IMAÑA-ENCINAS et al., 2009; RUFINI et al., 2010; MIRANDA et al., 2011; RIBEIRO et al., 2011).

A presença de espécies como C. leptophloeos com menores valores de biomassa seca acumulada como observado na pesquisa, pode ter influenciado no aumento do erro para biomassa total, fuste e galho fino + folhas, possivelmente explicado pelos seus baixos valores de densidade básica $(0,49$ $\left.\mathrm{g} / \mathrm{dm}^{3}\right)$ inferiores aos das demais espécies como; P. bracteosa $\left(0,82 \mathrm{~g} / \mathrm{dm}^{3}\right)$, M. ophthalmocentra $(1,06$ $\mathrm{g} / \mathrm{dm}^{3}$ ) e M. tenuiflora (1,03 g/dm³) (ALBUQUERQUE, 2015; SILVA et al., 2011). 


\section{CONCLUSÕES}

Os modelos ajustados de Schumacher-Hall e Gompertz, puderam gerar equações com baixos erros de predição para biomassa seca existente na região de estudo, pois as espécies abordadas representam cerca de 70\% da dominância e densidade nas áreas de caatinga arbustivo-arbórea em estágio de sucessão secundária intermediária, comumente encontradas no sertão de Pernambuco.

Desse modo, o uso de equações para predição de biomassa puderam facilitar a mensuração do carbono nestas áreas, visando reduzir a supressão e possibilitando o fomento ao uso dos créditos de carbono na região, como um viés econômico sustentável e lucrativo.

Outro ponto a ser observado foi que o trabalho gerou equações capazes de representar a biomassa seca de diferentes partes da planta, permitindo quantificar previamente a biomassa total a ser retirada ou deixada em campo como aporte necessário ao reestabelecimento nutricional do sítio garantindo a manutenção do mesmo, bem como a redução dos impactos causados nestas áreas já fragilmente perturbadas.

\section{REFERÊNCIAS BIBLIOGRÁFICAS}

ABREU, J. C. Modelagem de biomassa e de nutrientes de espécies da Caatinga no município de Floresta PE. 2012. 111 p. Dissertação (Mestrado em Ciências Florestais) - Universidade Federal Rural de Pernambuco, Recife, 2012.

ABREU, J. C.; SILVA, J. A. A.; FERREIRA, R. L. C.; ALVES JUNIOR, F. T. Ajuste de modelos matemáticos lineares e não lineares para estimativa de biomassa e nutrientes de Anadenanthera colubrina var. cebil no semiárido pernambucano. Scientia Florestalis, Piracicaba, v. 44, n. 111, p. 739-750, 2016. DOI: http://dx.doi. org/10.18671/scifor.v44n111.20

ALBUQUERQUE, C. S. Densidade de madeira de espécies arbustivas-arbóreas em diferentes cotas altitudinais da Serra de Bodocongó, Semiárido Brasileiro. 2015. 48 p. Monografia (Graduação em Ciência Biológicas) - Universidade Estadual da Paraíba, João Pessoa, 2015.

ALVES JUNIOR, F. T. Estrutura, biomassa e volumetria de uma área de Caatinga, Floresta-PE. 2010. 151 p. Tese (Doutorado em Ciências Florestais) - Universidade Federal Rural de Pernambuco, Recife, 2010.

AMORIM, A. M.; IARDIM, J. G.; CLIFTON, B. C.; FIASCHI, P.; THOMAS, W. W.; CARVALHO, A. M. V. The vascular plants of a forest fragment in southern Bahia, Brazil. Sida, v. 21, n. 3, p. 1726 - 1752, 2005.

ARAÚJO, B. A.; DANTAS NETO, J.; ALVES, A. S; ARAÚJO, P. A. A. Estrutura fitossociológica em uma Área de Caatinga no Seridó Paraibano. Revista Educação Agrícola Superior, Brasília, v. 27, n. 1, p. 25-29, 2012.

BASUKI, T. M.; VAN LAAKE, P. E.; SKIDMORE, A. K.; HUSSIN, Y. A. Allometric equations for estimating the above-ground biomass in tropical lowland Dipterocarp forests. Forest Ecology and Management, Amsterdan, v. 257, n.8, p.1684-1694, 2009. DOI: http://dx.doi.org/10.1016/i.foreco.2009.01.027

BOX, G. E. P.; COX, D. R. An analysis of transformations. Journal of the Royal Statistical Society. Series B (Methodological), London, v. 26, n. 2, p. 211-252, 1964.

BRAUN-BLANQUET, J. Plant sociology: the study of plant communities. New York: McGraw-Hill, 1932. 438 p.

CABRAL, G. A. DE L.; SAMPAIO, E. V. DE S. B.; ALMEIDA-CORTEZ, J. S. DE. Estrutura Espacial e Biomassa da Parte Aérea em Diferentes Estádios Sucessionais de Caatinga, em Santa Terezinha, Paraíba. Revista Brasileira de Geografia Física, Recife, v. 6, n. 3, p. 566-574, 2013.

CALIXTO JÚNIOR, J. T.; DRUMOND, M. A. Estrutura Fitossociológica de um Fragmento de Caatinga Sen- Su Stricto 30 anos após Corte Raso, Petrolina-PE, Brasil. Revista Caatinga, Mossoró, v. 24, n. 2, p. 67-74, 2011.

Sci. For., Piracicaba, v. 46, n. 118, p. 285-295, jun. 2018 DOI: dx.doi.org/10.18671/scifor.v46n118.14 
Barreto et al. - Ajuste de modelos matemáticos à biomassa seca

dos compartimentos de plantas lenhosas em área de caatinga

CASTELLETTI, C. H. M.; CARDOSO, J. M. S.; TABARELLI, M.; MELO, A. M. S. Quanto ainda resta da Caatinga? Uma estimativa preliminar. In: TABARELLI, M.; CARDOSO, J. M. S. Biodiversidade da Caatinga: áreas e ações prioritárias para a conservação. Recife: MMA / UFPE, 2004. p. 91-100.

CAVALCANTI, E. R.; ARAÚJO. N. C. F. O uso da energia de biomassa no Bioma Caatinga. In: SEMANA DO MEIO AMBIENTE. 5., Recife, 2008. Resumos... Recife: Fundaj, 2015. Disponível em:< http://www.fundaj.gov. br/geral/VSMA/VSMA2008 2302.pdf >. Acesso em: 15 maio 2015.

COELHO, M. S.; ALMADA, E. D.; QUINTINO, A. V.; FERNANDES, G. W.; SANTOS, R. M.; SÁNCHEZ-AZOFEIFA, A.; SANTO, M. M. D. Floristic composition and structure of a tropical dry forest at different successional stages in the Espinhaço Mountains, southeastern Brazil. Interciencia, Caracas, v. 37, n. 3, p. 190-196, 2012.

CPRH - AGÊNCIA ESTADUAL DE MEIO AMBIENTE. Instrução Normativa CPRH No 07/2006 de 29 de dezembro de 2006. Recife, 2006. Disponível em: < http://www.cprh.pe.gov.br >. Acesso em: 07 set. 2016.

CPRM - SERVIÇO GEOLÓGICO DO BRASIL. Diagnóstico do município de Floresta. Estado de Pernambuco. Pernambuco: Serviço Geológico do Brasil, 2005. Disponível em: < http://www.cprm.gov.br/rehi/atlas/ pernambuco/relatorio/FLOR061.pdf >. Acesso em: 12 out. 2016.

DRUMOND, M. A.; PIRES, I. E.; OLIVEIRA, V. R.; OLIVEIRA, A. R. ALVAREZ, I. A. Produção e distribuição de biomassa de espécies arbóreas no semiárido brasileiro. Revista Árvore, Viçosa, v.32, n.4, p. 665-669, 2008. DOI: http://dx.doi.org/10.1590/S0100-67622008000400007

EMBRAPA - EMPRESA BRASILEIRA DE PESQUISA AGROPECUÁRIA. Zoneamento agroecológico do estado de Pernambuco - ZAPE. 2001. Disponível em:< http://www.uep.cnps.embrapa.br/zape/cartas/Airi.pdf >. Acesso em: 12 out. 2016.

FABRICANTE, J. R.; FEITOSA, S. S.; BEZERRA, F. T. C.; FEITOSA, R. C.; XAVIER, K. R. F. Análise populacional de Caesalpinia pyramidalis Tul. (Fabaceae Lindl.) na caatinga da região do Seridó nordestino. Revista Brasileira de Biociências, Porto Alegre, v. 7, n. 3, p. 285-290, 2009.

FERRAZ, J. S. F.; FERREIRA, R. L. C.; SILVA, J. A. A.; MENUER, M. J.; SANTOS, M. V. F. Estrutura do componente arbustivo-arbóreo da vegetação em duas Áreas de Caatinga, no Município de Floresta, Pernambuco, Brasil. Revista Árvore, Viçosa, v. 38, n. 6, p. 1055-1064, 2014.

FERREIRA, R. L. C.; SILVA, S. O.; SILVA, J. A. A.; LIRA, M. A.; ALVES JÚNIOR, F. T.; NASCIMENTO, L. M. Richness and diversity of Caatinga áreas in diferente sucessional stages in northeastern Brazil. Scientia Forestalis, Piracicaba, v. 44, n. 112, p.799-810, 2016. DOI: http://dx.doi.org/10.18671/scifor.v44n112.02

GIULIETTI, A. M.; NETA, A. L. B.; CASTRO, A, A. J. F.; GAMARRA-ROJAS, C. F. L.; SAMPAIO, E. V. S.; VIRGÍNIO, J. F.; QUEIROZ, L. P.; FIGUEIREDO, M. A.; RODAL, M. J. N.; BARBOSA, M. R. V.; HARLEY, R. M. Diagnóstico da vegetação nativa do bioma Caatinga: Biodiversidade da Caatinga: áreas e ações prioritárias para a conservação, Brasília: MMA, 2004. 90 p, 2004.

IMAÑA-ENCINAS, J.; SANTANA, O. A.; DE PAULA, J. E.; IMAÑA, C. R. Equações de volume de madeira para o cerrado de Planaltina de Goiás. Revista Floresta, Curitiba, v. 39, n. 1, p. 107-116, 2009.

LEAL, I. R.; SILVA, J. M.; TABARELLI, M.; LACHER JÚNIOR, T. Mudando o curso da conservação da biodiversidade na Caatinga do Nordeste do Brasil. Megadiversidade, v. 1, n. 1, p. 139-146, 2005.

LIMA, P. C. F.; OLIVEIRA, E. B.; MACHADO, S. A. Equações para estimativa da biomassa de espécies de Prosopis no semi-árido brasileiro. Boletim de Pesquisa Florestal, Colombo, v. 32/33, n. 1, p. 67-79, 1996.

MARANGON, G. P.; FERREIRA, R. L. C.; SILVA, J. A. A.; LIRA, D. F. S.; SILVA, E. A.; LOUREIRO, G. H. Estrutura e padrão espacial da vegetação em uma área de caatinga. Floresta, Curitiba, v. 43, n. 1, p. 83-92, 2013. 
MIRANDA, D. L. C; MELO, A. C. G.; SANQUETTA, C. R. Equações alométricas para estimativa de biomassa e carbono em árvores de reflorestamento de restauração. Revista Árvore, Viçosa, v. 35, n. 3, p. 679-689, 2011. http://dx.doi.org/10.1590/S0100-67622011000400012

MUELLER-DOMBOIS, D.; ELLEMBERG. H. Aims and methods of vegetation ecology. New york: John Wiley \& Sons, 1974. 547 p.

REZENDE, A. V.; VALE, A. T.; SANQUETTA, C. R.; FIGUEIREDO FILHO, A.; FELFILI, J. M. Comparação de modelos matemáticos para estimativa do volume, biomassa e estoque de carbono da vegetação lenhosa de um cerrado sensu stricto em Brasília, DF. Scientia Forestalis, Piracicaba, n. 71, p. 65-76, 2006.

RIBEIRO, S. C.; FERHRMANN, L.; SOARES, C. P. B.; JACOVINE, A. G.; KLEINN, C.; GASPAR, R. O. Above- and belowground biomass in a Brazilian Cerrado. Forest Ecology and Management, Amsterdan, v. 262, n. 3, p. 491-499, 2011. DOI: http://dx.doi.org/10.1016/j.foreco.2011.04.017

RIEGELHAUPT, E. M; PAREYN, F. G. C. A questão energética e o manejo florestal da Caatinga. In: GARIGLIO, M. A; SAMPAIO, E. V. S. A; CESTARO, L. A; KAGEYAMA, P. Y. (Org.). Uso Sustentável e conservação dos recursos florestais da Caatinga, Brasília: Serviço Florestal Brasiliero, 2010. p.65-75.

RUFINI, A. L.; SCOLFORO, J. R. S.; OLIVEIRA, A. D.; MELLO, J. M. Equações volumétricas para o cerrado Sensu Stricto, em Minas Gerais. Cerne, Lavras, v. 16, n. 1, p. 1-11, 2010. DOI: http://dx.doi.org/10.1590/ $\underline{\text { S0104-77602010000100001 }}$

SAMPAIO, E. V. S. B.; SILVA, G. C. Biomass equations for Brazilian semiarid caatinga plants. Acta Botanica Brasilica, v. 19, n. 4, p. 937-945, 2005. DOI: http://dx.doi.org/10.1590/S0102-33062005000400028

SANTANA, J. A. S.; SOUTO, J. S. Diversidade e estrutura fitossociológica da caatinga na estação ecológica do Seridó, RN. Revista de Biologia e Ciências da Terra, Campina Grande, v. 6, n. 2, p. 232-242, 2006.SANTOS, K.; SANQUETA, C. R. EISFIELD, R. L. WATZLAWICK, L. F.; ZILIOTTO, M. A. B. Equações volumétricas por classe diamétrica para algumas espécies folhosas da Floresta Ombrófila Mista no Paraná, Brasil. Revista Ciências Exatas e Naturais, Guarapuava, v. 8, n. 1, p. 99-112, 2006.

SCHUMACHER, F. X.; HALL, F. S. Logaritmic expression of timber volume. Journal of Forestry, Bethesda, v.37, n.9, p.719-734, 1939.

SILVA, G. C.; SAMPAIO, E. V. S. B. Biomassas de partes aéreas em plantas da caatinga. Revista Árvore, Viçosa, v. 32, n. 3, p. 567-575, 2008. DOI: http://dx.doi.org/10.1590/S0100-67622008000300017

SILVA, L. B.; SANTOS, F. A. R.; GASSON, P.; CUTLER. Comparative study of Mimosa ophthalmocentra Mart. ex Benth and Mimosa tenuiflora (Willd.) Poir.(Fabaceae-Mimosoideae) wood in the caatinga of Northeast Brazil. Acta Botanica Brasilica, v. 25, n. 2, p. 301-314, 2011. DOI: http://dx.doi.org/10.1590/S0102$\underline{33062011000200006}$

SILVA, M. L. M.; BINOTI, D. H. B.; GLERIANI, J. M.; LEITE, H. G. Ajuste do modelo de Schumacher e Hall e aplicação de redes neurais artificiais para estimar volume de árvores de Eucalipto. Revista Árvore, Viçosa, v.33, no .6, p.1133-1139, 2009. DOI: http://dx.doi.org/10.1590/S0100-67622009000600015

ZÁKIA, M. J. B.; PAREYN, F. G.; RIEGELHAUPT, E. Equações de peso e de volume para oito espécies lenhosa nativas do Seridó, RN. In: IBAMA. Plano de manejo florestal para a região do Seridó do Rio Grande do Norte. Natal: PNUD / FAO / IBAMA - Governo do Rio Grande do Norte, 1992. p. 1-92.

Recebido em 08/02/2017

Aceito em 18/08/2017 
